\title{
What is the role of extracorporeal membrane oxygenation for hypoxic failure during an influenza pandemic?
}

\author{
Andrew J. Baker, MD
}

Published online: 9 January 2010

(C) Canadian Anesthesiologists' Society 2010

Many of us have participated in the planning for disasters and pandemics at the hospitals where we practice. And for many this has included system level plans for surge capacity. Anesthesiologists and critical care physicians are acutely aware of issues of system capacity in general. The recent experiences with influenza A (H1N1) 2009 globally have caused many of us to think about our system capacity and to do mental experiments imagining different scenarios of demand and supply ratios for critical care capacity. When these ratios are imagined in the extreme, the value of primary prevention quickly becomes paramount.

However, much of our practice is about how to respond when primary prevention fails or is insufficient. Typically, while we try to steer our populations and patients in a healthier direction, we develop a strategy to deal at each juncture with the worse scenario. In the context of disasters and pandemics we must ask the questions: what is it that might be required at each of the junctures, and what is our capacity to provide it? It is the intersection of these two questions that creates the difficulties. The capacity obviously depends on what we are asked to provide; and in turn what we can provide depends on the capacity required. Nevertheless the history of modern critical care is the process of building capacity.

When planning for a pandemic, the natural history of the disease makes critical differences. Modeling the demand for different types of resources is essential for planning capacity. For influenza pandemics that migrate across the world, there is the advantage for some countries of a

\section{A. J. Baker, MD ( $\square)$}

Department of Critical Care Medicine and Anesthesia,

St. Michael's Hospital, 30 Bond Street, 4010 Bond Wing,

Toronto, Ontario M5B 1W8, Canada

e-mail: bakera@smh.toronto.on.ca preview of what to expect from the prior experience of others. ${ }^{1-5}$ Of critical importance however is the development of standardized case report forms so that the natural history of the illness can be compared and even tracked as it evolves. In this regard, Canada has played an important role in developing a standardized case reporting methodology that has been used to compare the disease internationally. ${ }^{2,6}$

At a population level influenza is characterized by a pyramid where very large numbers of people may be exposed, but the severity of illness worsens in exponentially fewer people. Not surprisingly the approach from a public health perspective is to focus on reducing exposure and on immunization. When the illness strikes, therapy for the most part is home-based supportive measures. The most compelling questions arising from influenza A (H1N1) 2009 have been about the determinants of this pyramid of severity. What is the ratio of those with influenza who need hospitalization? What are the co-morbid, phenotypic or genotypic risk factors? Why does it progress so rapidly and so severely in some people? And practically, what are effective treatment or support options in the more severely affected? In this last regard another innovation, in which Canada is actively involved in the leadership, is the International Forum for Acute Care Trialists (InFACT) group - a global consortium of organizations aiming to undertake large scale interventional trials with agents that are simple to use and approved already. ${ }^{7}$ Some of the questions include the dosing of existing antiviral agents, or the utility of steroids in reducing the severity of adult respiratory distress syndrome.

People who have the most severe manifestation of the illness have died from rapidly evolving multiple organ failure including severe adult respiratory distress syndrome. The approaches to severe hypoxic respiratory 
failure include optimizing the balance of oxygen demand and delivery, advanced conventional ventilation techniques, extracorporeal lung support and specialized ventilation such as high frequency oscillation (HFO). With respect to this influenza and future pandemics, these approaches to hypoxic failure raise the obvious questions: Do they work? If so, in whom do they work? The answers to these questions lead us back to our pyramid and our original questions about capacity and feasibility.

There have been a number of important recent publications which will help us triangulate the answers to these questions. In this issue of the Journal, Freed et al. present the characteristics of six patients who received extracorporeal lung support and who had severe respiratory failure secondary to influenza A (H1N1) 2009 infection in Canada. ${ }^{8}$ This group represented $3.6 \%$ of 168 patients with influenza A (H1N1) 2009 in Canada who were critically ill between April and August 2009 and were part of a prospective observational study reported in JAMA November 4 $2009 .{ }^{6}$ At the same time an Australasian group reported on 68 patients who received extracorporeal membrane oxygenation (ECMO) for acute respiratory distress syndrome arising from influenza A (H1N1) 2009 infection and who represented $33 \%$ of 201 critically ill patients with H1N1. ${ }^{9}$

These print publication dates were only weeks following that of the Conventional Ventilation or ECMO for Severe Adult Respiratory Failure (CESAR) trial in the Lancet which assessed the efficacy and economic value of conventional ventilatory support compared with ECMO for severe adult respiratory failure. ${ }^{10}$ In this study 180 patients were included, 90 assigned to referral for treatment by ECMO and 68 received it. This study is the subject of a Best Evidence in Critical Care Medicine review in this issue of the Journal. ${ }^{11}$ Also, just after the publication of the CESAR trial, Brogan et al. reported on a retrospective case review of 1473 patients receiving ECMO for acute respiratory failure from $1986-2006 .^{12}$

How do we incorporate all of this information into a strategy for patient and population care? The various case series, such as Freed et al.'s in this month's issue, help us understand the details of how ECMO was used, and how it may have worked for certain patients. However, one is drawn to the question of not whether ECMO is useful, but for whom does it work. After this is better understood, and in conjunction with what we learn about the pyramid of the disease, pandemic planners and clinicians must work together to understand the capacity and feasibility issues. The Australasian experiences demonstrated the ability of certain centres and regions to handle relatively large numbers of cases of ECMO simultaneously as well as the rates and severity of complications. The CESAR trial demonstrated that even centres of excellence during a trial can face rate limiting capacity issues and that we must titrate carefully the inclusion criteria in order to demonstrate usefulness.

The struggle to understand the role of ECMO, the rapidity with which some strains of influenza can progress to life threatening lung and organ failure, and the widely fluctuating estimates of demand supply for critical care and intensive salvage techniques highlight the importance of the need for multiple perspectives on this problem. Prevention, containment and immunization will have a definite failure rate. Research and development of treatments aimed at preventing severity escalation, such as those pursued by InFACT, is imperative. Resource-intensive salvage techniques such as ECMO must be developed in conjunction with understanding their role. This includes focusing our questions on: identifying accurately for whom this technique is most valuable, what its value relative to alternatives (HFO for example) is, and how we mitigate or at least integrate the complication rate of ECMO into this complex calculation. Finally, the question of anticipating capacity needs, developing capacity and identifying the patient inclusion criteria are critical, but there is also the challenge of sequential triage. The concept of triage originates from situations where the initial task is to assess the scope and scale of the problem. Sequential triage occurs when the disaster unfolds over time and despite best efforts, estimates of demand-capacity change and evolve over time. Setting thresholds for intervention based on these ratios set against the likelihood that an intervention will be useful is significantly more complex in practice when triage is sequential.

Finally, it is imperative that the experience of each pandemic is captured accurately. The tradition of medicine is based in the stepwise progress made by the careful observations of disasters and pandemics and by pushing boundaries with innovative technology. The two articles in this issue of the Journal continue this tradition; however there is still insufficient data to understand fully the role of ECMO in hypoxic failure during an influenza pandemic. Nevertheless, it remains our job to integrate the available information into the challenges that no doubt face us in the future.

\section{Quel rôle joue l'oxygénation par une membrane extracorporelle dans le traitement de la défaillance hypoxique pendant une pandémie de grippe?}

Nous sommes nombreux à avoir pris part à des comités de planification en cas de catastrophe et de pandémie dans les institutions où nous pratiquons. Dans plusieurs cas, ces 
planifications ont aussi inclus des projets visant à augmenter la capacité du système en cas d'urgence. Les anesthésiologistes et intensivistes sont en général très sensibles aux questions de capacité du système. Les expériences récentes liées à la grippe A (H1N1) 2009 à l'échelle planétaire ont incité beaucoup d'entre nous à réfléchir à la capacité de notre système et à imaginer différents scénarios de ratios offre/demande par rapport à nos capacités en matière de soins critiques. Si nous imaginons le pire, la valeur de la prévention primaire prend immédiatement le devant de la scène.

Et pourtant, une grande partie de notre pratique porte sur la façon de réagir lorsque la prévention primaire échoue ou s'avère insuffisante. En règle générale, nous élaborons une stratégie pour être capable à tout instant de faire face au pire scénario tout en orientant nos populations et nos patients dans une direction plus saine. Dans le cas de catastrophes et de pandémies, les questions suivantes doivent être posées: de quoi pourrait-on avoir besoin à chaque instant, et dans quelle mesure nos capacités nous permettent-elles de le fournir? Mais les difficultés surviennent là où ces deux questions se rencontrent. Les capacités dépendent évidemment de ce qu'on nous demande de fournir; de la même manière, ce que nous pouvons fournir dépend des capacités requises. Nonobstant, le processus de création de capacités est l'histoire même des soins critiques modernes.

Lors des préparatifs pour faire face à une pandémie, l'histoire naturelle de la maladie joue un rôle crucial et change considérablement la donne. Il est essentiel de façonner la demande pour différents types de ressources lors de la planification des capacités. Lors de pandémies de grippe se répandant aux quatre coins de la planète, certains pays ont l'avantage d'avoir un aperçu de ce à quoi ils devront faire face grâce à l'expérience d'autres. ${ }^{1-5}$ Toutefois, l'élaboration de formulaires de présentations de cas standardisés est cruciale de façon à pouvoir comparer l'histoire naturelle de la maladie, et même à pouvoir la suivre au fil de son évolution. Le Canada a, à cet égard, joué un rôle important dans la mise au point d'une méthodologie standardisée de présentation de cas, laquelle a été utilisée pour comparer la maladie sur le plan international. $^{2,6}$

$\mathrm{Au}$ niveau de la population, la grippe peut être représentée sous forme de pyramide: un nombre très important de personnes pourrait être exposé à la maladie, mais sa gravité empire dans un nombre exponentiellement moindre de personnes. Il est dès lors peu surprenant que l'approche à adopter, du point de vue de la santé publique, est de se concentrer sur la réduction de l'exposition et sur l'immunisation. Lorsque la maladie frappe, le traitement consiste principalement en des mesures de soutien à prendre à la maison. Les questions les plus pressantes à avoir été posées au sujet de la grippe A (H1N1) 2009 concernent les éléments déterminants de cette pyramide de gravité. Quelle est la proportion des personnes ayant contracté la grippe qui nécessitent une hospitalisation? Quels sont les facteurs de risque de comorbidité, de phénotypes et de génotypes? Pourquoi progresse-t-elle si rapidement et de façon si grave chez certaines personnes? Et, d'un point de vue pratique, quelles sont les options de traitement ou de soutien efficaces pour les populations les plus gravement touchées? À ce propos, le Canada se trouve à nouveau à la tête de l'innovation avec d'autres pays: le groupe «International Forum for Acute Care Trialists» (InFACT) est un consortium international d'organismes dont le but est de réaliser des études interventionnelles d'envergure avec des agents à la fois faciles à utiliser et déjà approuvés par les organismes de réglementation. ${ }^{7}$ Parmi les questions abordées, citons le dosage des agents antiviraux existants, ou encore l'utilité des stéroïdes pour réduire la gravité du syndrome de détresse respiratoire chez l'adulte.

Les personnes présentant les manifestations les plus graves de la maladie sont décédées à la suite de défaillance multisystémique progressant rapidement, y compris d'un syndrome de détresse respiratoire grave chez l'adulte. Parmi les différentes approches dont nous disposons pour traiter une détresse respiratoire hypoxique grave figurent l'optimisation de l'équilibre entre les besoins et l'apport en oxygène, les techniques avancées de ventilation traditionnelle, l'assistance pulmonaire extracorporelle et les méthodes de ventilation spécialisées, comme par exemple la ventilation à haute fréquence (VHF). En ce qui touche à cette grippe et aux pandémies futures, les différentes approches pour traiter la défaillance hypoxique soulèvent d'évidentes questions: sont-elles efficaces? si oui, dans quelle population? Les réponses à ces questions nous ramènent à notre pyramide et à nos questions originales quant aux capacités et la faisabilité.

Plusieurs publications récentes d'importance vont nous aider à déduire les réponses à ces questions. Dans ce numéro du Journal, Freed et coll. présentent les caractéristiques de six patients ayant reçu une assistance pulmonaire extracorporelle et ayant été victimes d'une détresse respiratoire grave à la suite d'une infection à la grippe A (H1N1) 2009 au Canada. ${ }^{8}$ Ce groupe représente 3,6\% des 168 patients souffrant de grippe A (H1N1)2009 au Canada à avoir été gravement malades entre avril et août 2009 et à avoir participé à une étude observationnelle prospective rapportée dans le JAMA du 4 novembre 2009 . $^{6}$ Au même moment, un groupe de chercheurs australasiens publiait un rapport portant sur 68 patients ayant reçu une oxygénation extracorporelle (ECMO) pour traiter un syndrome de détresse respiratoire aiguë provoqué par une 
infection à la grippe A (H1N1) 2009 et représentant $33 \%$ de 201 patients gravement malades de la H1N1. ${ }^{9}$

Les dates de publication de ces études suivaient de quelques semaines seulement celle de l'étude «Conventional Ventilation or ECMO for Severe Adult Respiratory Failure» (CESAR) dans The Lancet, qui évaluait l'efficacité et la valeur économique de l'assistance ventilatoire traditionnelle par rapport à l'ECMO pour traiter la détresse respiratoire grave chez l'adulte. ${ }^{10}$ Cent quatre-vingts patients ont participé à cette étude, dont 90 ont été référés pour être traités par ECMO, et 68 de ces patients ont reçu ce traitement. Cette étude fait l'objet d'un compte-rendu des Meilleures données probantes en soins intensifs dans ce numéro du Journal. ${ }^{11}$ À cela s'ajoute la publication, immédiatement après celle de l'étude CESAR, d'un rapport de Brogan et coll. portant sur une étude de cas rétrospective de 1473 patients ayant reçu l'ECMO pour traiter un syndrome de détresse respiratoire aiguë entre 1986 et $2006 .^{12}$

Dès lors, comment peut-on intégrer toutes ces informations de façon à élaborer une stratégie de soins pour les patients et la population? Les différentes séries de cas, telles celle de Freed et coll. dans ce numéro, nous aident à saisir en détail la manière dont l'ECMO a été utilisée et la façon dont elle aurait pu être efficace pour certains patients. Toutefois, la question que nous nous posons n'est pas de savoir si l'ECMO peut être utile, mais plutôt pour qui elle est efficace. Une fois que nous aurons mieux compris cela, et de pair avec ce que nous apprendrons au sujet de la pyramide de la maladie, les planificateurs et les cliniciens impliqués dans la planification des soins pour la pandémie devront travailler de concert afin de pouvoir répondre aux questions de capacités et de faisabilité. Les expériences australasiennes ont démontré la capacité de certains centres et régions à gérer un nombre relativement élevé d'ECMO simultanément ainsi que les taux et la gravité des complications. Dans l'étude CESAR, on a démontré que même des centres d'excellence peuvent être confrontés à des problèmes de capacités limitées; dès lors, il nous faut doser soigneusement les critères d'inclusion afin de démontrer l'utilité d'un traitement.

La lutte pour comprendre le rôle de l'ECMO, la rapidité avec laquelle certaines souches de grippe évoluent vers une défaillance pulmonaire ou systémique potentiellement mortelle et les estimations très fluctuantes d'offre et de demande de technique de sauvetage en soins critiques et intensifs-tous ces facteurs soulignent encore plus l'importance d'avoir plusieurs perspectives face à ce problème. La prévention, le confinement et l'immunisation se solderont par un certain taux d'échec. La recherche et le développement de traitements pour prévenir l'intensification de la gravité de la maladie, comme ceux sur lesquels le groupe InFACT travaille, sont essentiels.
Les techniques de sauvetage nécessitant beaucoup de ressources comme l'ECMO doivent être mises au point au fur et à mesure que progresse notre compréhension de leur rôle. Pour ce faire, nos questions doivent se concentrer sur l'identification précise de la population pour laquelle cette technique est le plus efficace, sa valeur par rapport aux alternatives (par ex. la VHF), et la façon de limiter ou pour le moins de prendre en compte le taux de complications liées à l'ECMO dans ce calcul complexe. Enfin, les questions d'anticipation des besoins en capacités, d'augmentation des capacités et d'identification des critères d'inclusion de patients sont elles aussi cruciales, sans oublier le défi d'un triage séquentiel. À l'origine, le concept de triage provient de situations où la tâche initiale est d'évaluer la portée et l'envergure du problème. On parle de triage séquentiel lorsque la catastrophe se déploie au fil du temps et, en dépit de tous les efforts, les estimations concernant la capacité et la demande changent et évoluent. La détermination de seuils d'intervention fondés sur ces ratios en regard de la probabilité qu'une intervention sera utile est bien plus compliquée dans la pratique lorsque le triage est séquentiel.

Enfin, il est impératif que les expériences liées à chaque pandémie soient saisies de façon exacte. La tradition médicale est basée sur les progrès accomplis au fil du temps grâce à une observation minutieuse des catastrophes et des pandémies et au dépassement des limites par des technologies innovantes. Les deux articles dans ce numéro du Journal s'inscrivent dans cette tradition; toutefois, nous ne disposons pas encore de suffisamment de données pour comprendre pleinement le rôle que joue l'ECMO dans la défaillance hypoxique pendant une pandémie de grippe. Notre rôle est cependant d'intégrer les informations à notre disposition dans le défi auquel nous aurons certainement à faire face à l'avenir.

Competing interests None declared.

\section{References}

1. White DB, Angus DC. Preparing for the sickest patients with 2009 influenza A (H1N1). JAMA 2009; 302: 1905-6.

2. Dominguez-Cherit G, Lapinsky SE, Macias AE, et al. Critically ill patients with 2009 influenza A(H1N1) in Mexico. JAMA 2009; 302: 1880-7.

3. Jain S, Kamimoto L, Bramley AM, et al. Hospitalized patients with 2009 H1N1 influenza in the United States, April-June 2009. N Engl J Med 2009; 361: 1935-44.

4. Wenzel RP, Edmond MB. Preparing for 2009 H1N1 influenza. N Engl J Med 2009; 361: 1991-3.

5. Influenza Investigators ANZIC, Webb SA, Pettila $V$, et al. Critical care services and 2009 H1N1 influenza in Australia and New Zealand. N Engl J Med 2009; 361: 1925-34.

6. Kumar A, Zarychanski R, Pinto $R$, et al. Critically ill patients with 2009 influenza A(H1N1) infection in Canada. JAMA 2009; 302: 1872-9. 
7. Marshall J. InFACT: a global critical care research response to H1N1. Lancet 2009. DOI:10.1016/S0140-6736(09)61792-x.

8. Freed DH, Henzler D, White $C W$, et al. Extracorporeal lung support for patients who had severe respiratory failure secondary to influenza A (H1N1) 2009 infection Canada. Can J Anesth 2010; 57(3).

9. Australia and New Zealand Extracorporeal Membrane Oxygenation (ANZ ECMO) Influenza Investigators, Davies A, Jones D, et al. Extracorporeal membrane oxygenation for 2009 influenza $\mathrm{A}(\mathrm{H} 1 \mathrm{~N} 1)$ acute respiratory distress syndrome. JAMA 2009; 302: 1888-95.

10. Peek GJ, Mugford M, Tiruvoipati R, et al. Efficacy and economic assessment of conventional ventilatory support versus extracorporeal membrane oxygenation for severe adult respiratory failure (CESAR): a multicentre randomised controlled trial. Lancet 2009; 374: 1351-63.

11. Brindley $P$, Cave D, Lequier L. Extracorporeal membrane oxygenation (ECMO) in severe adult respiratory distress syndrome. Can J Anesth 2010; 57(3).

12. Brogan T, Thiagarajan $R$, Rycus $P$, et al. Extracorporeal membrane oxygenation in adults with severe respiratory failure: a multi-center database. Intensive care medicine 2009. DOI: 10.1007/s00134-009-1661-7. 